\title{
Postbuckling strength of slender elliptical hollow sections in compression
}

\author{
F. McCann \\ London South Bank University, London, UK \\ C. Fang \\ Tongji University, Shanghai, China \\ L. Gardner \\ Imperial College London, London, UK \\ N. Silvestre \\ Instituto Superior Técnico, Universidade de Lisboa, Lisbon, Portugal
}

\begin{abstract}
Numerical analysis of the local buckling behaviour and ultimate cross-sectional strength of tubular elliptical profiles in compression has been performed. After validating the model against previous experimental results, a parametric study comprising a total of 270 elliptical sections was conducted in order to examine the influence of cross-section aspect ratio, geometric imperfections and local slendernesses. The obtained ultimate capacities, load-deformation responses and failure modes are discussed. It was found that for lower cross-section aspect ratios the behaviour of the elliptical hollow sections (EHS) was similar to that of cylindrical shells across a number of metrics; as the aspect ratio increased, more plate-like stable postbuckling behaviour was observed. A design method has been proposed for Class 4 EHS members that reflects the reduction in capacity due to local buckling with increasing slenderness, but also recognises the improved postbuckling stability with increasing aspect ratio.
\end{abstract}

\section{INTRODUCTION}

The focus of the present investigation is on the behaviour and strength of slender steel EHS tubes under compression. Potential applications of such members include aesthetic lightweight cladding rails, mullion posts and concrete-filled steel tubes in composite construction (McCann et al. 2015). A number of the current range of hot-finished elliptical hollow sections (Steel Construction Institute 2013), which have been used in a range of structural applications, as outlined in Chan et al. (2010), are Class 4 (slender) in compression. Cold-formed elliptical sections are also produced from both structural carbon steel (Zhu \& Young 2012) and stainless steel (Theofanous et al. 2009), and are often of slender proportions.

Existing studies (Ruiz-Teran \& Gardner 2008, Kempner \& Chen 1964, Silvestre 2008, Silvestre \& Gardner 2011, Abela \& Gardner 2012, Law \& Gardner 2013) of elastic local buckling and postbuckling of elliptical hollow sections have found that, in contrast to CHS tubes, EHS tubes in compression can have stable postbuckling responses and may therefore be able to resist further load beyond the elastic buckling load. Previous numerical studies (Silvestre \& Gardner 2011) into the elastic local postbuckling behaviour of EHS columns led to the following conclusions: i) the maximum stress that a fully-elastic EHS tube with an aspect ratio $a / b \geq 2.0$ could carry is higher than its critical buckling stress $f_{\text {cr }}$ due to the stable postbuckling response, where $2 a$ and $2 b$ are the larger and smaller outer diameters of the EHS, respectively, as shown in Figure 1; ii) concentrated zones of compressive stress were located near the point of minimum radius of curvature (akin to the edges of simply-supported plates), while the zones of maximum radius of curvature experienced an approximately uniform and relatively low compressive stress level; iii) the imperfection sensitivity significantly decreased with increasing $a / b$, representing a transition from shell-type behaviour (imperfection sensitive) for EHS columns with low $a / b$ ratios to plate-type behaviour (imperfection insensitive) with increasing $a / b$ ratios.

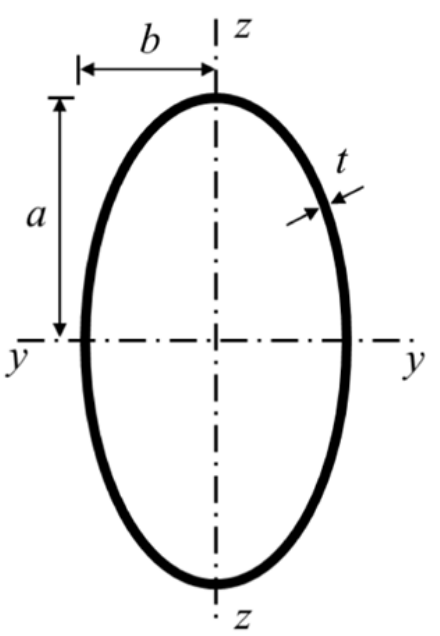

Figure 1. Cross-section of elliptical hollow section 
These observations suggest that strength curves for elliptical sections may need to be both a function of local slenderness to allow for the increased susceptibility to local buckling and cross-sectional aspect ratio $a / b$ to reflect the differing postbuckling stability.

In the present study, firstly, the development and validation of a numerical model to simulate the response of EHS in compression is described. After achieving satisfactory agreement between the numerical results generated herein and previous experimental results, the axial compressive response of EHS stub columns with aspect ratios ranging from 1.1 to 5.0 is examined. Other parameters varied in the study include local buckling slenderness and imperfection amplitude. The results of the parametric study are used as a basis for the formulation of new strength and effective area reduction curves for the design of Class 4 EHS compression members. Comparisons are made with existing provisions from $\mathrm{Eu}-$ rocode 3 for the design of CHS tubes, demonstrating the improved accuracy of the proposed design rules.

\section{DEVELOPMENT AND VALIDATION OF NUMERICAL MODEL}

In this section, the modelling strategy used to simulate the EHS stub columns in compression is described, followed by the validation of the model against previous experiments.

\subsection{Description of finite element model}

\subsubsection{Geometry}

A numerical model was developed using the finite element analysis software ABAQUS (ABAQUS 2012). Aspect ratios $a / b=1.1,1.5,2.0,3.0$ and 5.0 were considered. The reference geometry is based on the commercially-available $300 \times 150$ series of elliptical sections with $a / b=2.0$; the cross-sectional geometry for the other four aspect ratios was based on maintaining a constant perimeter $P$ of $726.3 \mathrm{~mm}$. In order to minimize length effects, a length effect coefficient proposed by Ruiz-Teran \& Gardner (2008) was used in the present study to define suitable lengths for the EHS models, while also precluding global buckling.

\subsubsection{Meshing}

In keeping with Silvestre \& Gardner (2011), the EHS tubes were meshed using 4-noded isoparametric reduced-integration S4R shell elements with a characteristic element size of $9 \mathrm{~mm}$.

\subsubsection{Boundary conditions}

Rigid plates, modelled using 3-noded R3D3 elements, were attached to the end sections of the stub columns using tie constraints. Fully-fixed boundary conditions were imposed on one end plate, while a compressive axial load was applied at the centroid of the other rigid end plate, on which all degrees-offreedom except longitudinal displacement were fixed.

\subsubsection{Analysis procedure}

Each simulation was conducted in two steps. The first step was a linear eigenvalue analysis, with the critical buckling stress $f_{\text {cr }}$ being that associated with the first valid buckling mode. This provided the initial imperfect geometry for the second step, a Riks arclength continuation analysis, which simulated the nonlinear behaviour of the EHS up to and beyond the ultimate load. The sensitivity of the system to initial imperfections was assessed by running simulations with three different imperfection amplitudes for each combination of cross-section and yield stress.

\subsubsection{Material modelling}

For the linear eigenvalue analyses, the stub column material was assumed to be homogeneous, isotropic and linearly-elastic with a Young's modulus of $216400 \mathrm{MPa}$ (Chan \& Gardner 2008a) and a Poisson's ratio of 0.3 . For the Riks analyses, elasticperfectly plastic material models were adopted, with three different yield stresses considered for each aspect ratio. No residual stresses were included in the numerical analyses since they have been found to be of very low magnitude in hot-finished elliptical tubes (Chan \& Gardner 2008a, b).

\subsubsection{Imperfections}

Appropriate imperfection shapes (Silvestre \& Gardner 2011) for each simulation were obtained from the linear eigenvalue analysis. The imperfection amplitudes $\Delta \mathrm{w}$ were calculated using an expression modified from clause D.1.2.2(1-2) of EN 19931-6 (CEN 2007) for circular shells for use with elliptical shells. The modified form of the expression is:

$\Delta w=\frac{t}{Q} \sqrt{\frac{r_{\mathrm{eq}}}{t}}$

where $r_{\mathrm{eq}}=\left(a^{2} / b\right)$ and $t$ are the equivalent radius and thickness of the elliptical shell, respectively, and $Q$ is a fabrication quality parameter. In the present study, three different levels of initial imperfection amplitude were considered, namely, $\Delta \mathrm{w}=0.1 t, Q=$ 40 (Class $\mathrm{A}$ - excellent quality), and $Q=25$ (Class $\mathrm{B}$ - high quality). Class A imperfections were found to provide an upper bound to measured values and can be assumed to represent a level of imperfection amplitude suitable for the design of hot-finished EHS (McCann et al. 2016). 


\subsection{Validation of numerical model}

Results from experiments by Chan \& Gardner (2008a) on hot-finished EHS stub columns were used to validate the numerical model. The three most slender cross-sections tested were selected for comparison: $150 \times 75 \times 4$ EHS, $400 \times 200 \times 8$ EHS and $500 \times 250 \times 8$, with the measured properties being used in the validation study. Good agreement between the experimental and numerical results was observed when considering the deformation mode (see Figure 2 ), initial stiffness, ultimate load $\left(P_{\mathrm{u}}\right)$, and general load-end shortening response, enabling an extensive parametric study to be conducted. When comparing the test results and the FE predictions for the ultimate load the maximum error of $3.9 \%$.
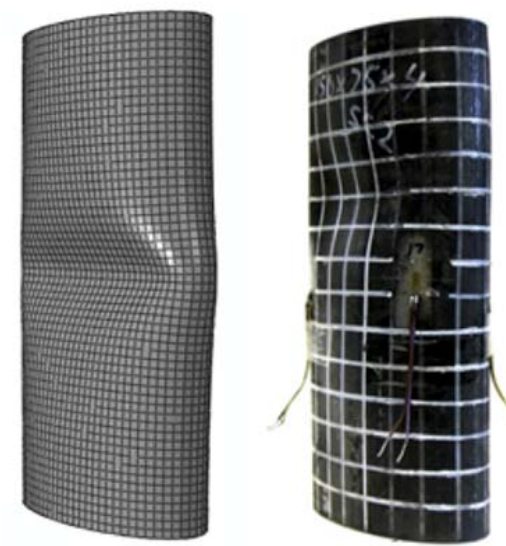

Figure 2. Comparison of numerical (left-hand side) and experimental (right-hand side) deformation modes

\section{NUMERICAL PARAMETRIC STUDY}

Having shown that good agreement exists between the predictions of the FE model and the experimental results of Chan \& Gardner (2008a) a parametric study was conducted that examined the influences of a number of key variables. In this section, the parameters varied in the study and the subsequent results obtained are presented and discussed.

\subsection{Parameters for numerical studies}

A range of nondimensionalised local buckling slendernesses $\bar{\lambda}_{\ell}$, as defined by Eq.(2), was considered by varying the thickness $t$ and yield strength $f_{\mathrm{y}}$ of the EHS across the five examined aspect ratios.

$\bar{\lambda}_{\ell}=\sqrt{\frac{f_{\mathrm{y}}}{f_{\mathrm{cr}}}}$

where $f_{\text {cr }}$ is the elastic critical local buckling stress,:

$f_{\text {cr }}=\frac{E}{\sqrt{3\left(1-v^{2}\right)}} \frac{2 t}{D_{\text {eq }}}$

where $D_{\text {eq }}=2 r_{\text {eq }}=2\left(a^{2} / b\right)$ is the equivalent diameter of the EHS under pure compression. Since the focus of this study is on the behaviour and strength of slender cross-sections, the selected EHS geometries were mainly Class 4. An elliptical hollow section can be classified as Class 4 if it satisfies the following condition (Gardner \& Chan 2007) based on the EN 1993-1-1 (CEN 2005) classification limits for CHS columns:

$\frac{D_{\text {eq }}}{t \varepsilon^{2}}=\frac{2\left(a^{2} / b\right) f_{\mathrm{y}}}{235 t}>90$

For $E=216400 \mathrm{MPa}$ (Chan \& Gardner 2008a) this corresponds to a local slenderness requirement for a Class 4 EHS of $\bar{\lambda}_{\ell}>0.284$, while for $E=210000$ $\mathrm{MPa}$, the corresponding limiting slenderness is 0.288 . In total, 270 cases (five aspect ratios $\times$ six thicknesses $\times$ three yield strengths $\times$ three imperfection classes) were simulated in the parametric study, the details of which are fully summarized in McCann et al. (2016).

\subsection{Failure modes}

The four deformation modes identified by Insausti \& Gardner (2011) were observed upon examination of the results of the parametric study: i) the shell-like "elephant foot" (EF) mechanism which is more prevalent for small imperfection amplitudes, with outward bulges forming a concertina; ii) the shelllike Yoshimura (Y) mechanism, with sequential folding at mid-height, which occurs for imperfection modes with inward displacements at mid-height; iii) the plate-like flip disc (FD) and iv) split flip disc (SFD) mechanisms, which are inward-facing with two parabolic hinge lines folding inwards and outwards, respectively (Murray 1984). In general, for the lowest aspect ratios $(a / b=1.1$ and 1.5$)$, the most prevalent failure mode tended to be the EF mode, with a superposition of FD and EF modes becoming more apparent as slenderness increased. With increasing aspect ratio, the Y mode became more obvious, with a superposition of $\mathrm{Y}$ and FD modes being the dominating failure mode for EHS with $a / b=$ 5.0. Further discussion of the failure modes can be found in McCann et al. (2016).

\subsection{Load-displacement behaviour}

Examples of load-end shortening curves obtained from the FE models are presented in normalised form $\left(f / f_{\mathrm{cr}} \mathrm{vs} . e / e_{\mathrm{cr}}\right.$, where $e$ is the end shortening and $e_{\mathrm{cr}}$ is the end shortening at the elastic buckling stress $f_{\text {cr }}$ obtained from a linear eigenvalue analysis) in Figure 3. Curves for intermediate slendernesses are plotted for $a / b=1.1,2.0$ and 5.0, where it can be seen that the stability of the postbuckling response increases with increasing aspect ratio. For $a / b=1.1$, there is cylindrical shell-like unstable postbuckling behaviour and considerable imperfection sensitivity. For $a / b=2.0$, the postbuckling response is somewhat stronger with less imperfection sensitivity, 
while for $a / b=5.0$, the behaviour is closer to that of a flat plate with a stable postbuckling equilibrium path and negligible imperfection sensitivity. Further discussion is provided in McCann et al. (2016) but in summary, the overall trends that can be observed from the load-displacement graphs are: i) increasing stability of the postbuckling response, and thus greater normalised load-carrying capacity, with increasing aspect ratio; ii) increasing stability of the postbuckling response with increasing local slenderness; iii) decreasing imperfection sensitivity with increasing slenderness. It is the aim of the present study to propose design curves (see Section 4) appropriately so that these trends are reflected.
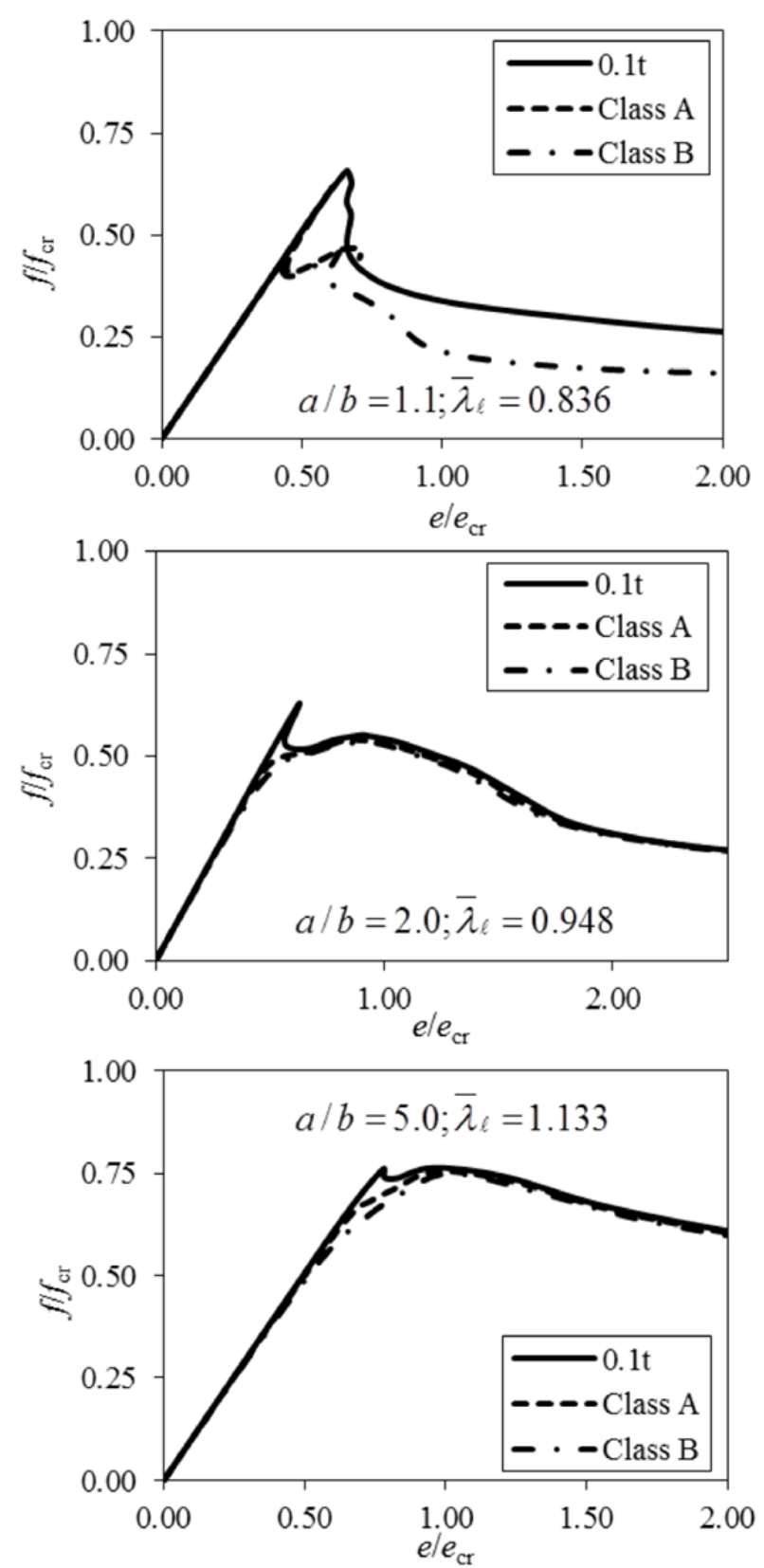

Figure 3. Load-end shortening curves for different aspect ratios

\subsection{Strength reduction}

The reduction in strength of the EHS stub columns is characterised by the ultimate-to-yield stress ratio $\rho=f_{\mathrm{u}} / f_{\mathrm{y}}$, or in terms of loads, $\rho=P_{\mathrm{u}} / P_{\mathrm{y}}$, where
$P_{\mathrm{u}}$ is the maximum load obtained by the stub columns and $P_{\mathrm{y}}$ is the yield load. Owing to the redistribution of compressive stresses from areas where local buckling is the most severe towards the areas of the elliptical sections with higher local curvature, the concept of a loss of effectiveness can be adopted as the basic design approach. Thus, the effective area $A_{\text {eff }}$ of the elliptical section can be obtained from:

$\rho=\frac{f_{\mathrm{u}}}{f_{\mathrm{y}}}=\frac{A_{\mathrm{eff}}}{A}$

When considering perfect elastic buckling, the buckling curve is given by:

$\rho=\frac{1}{\bar{\lambda}_{\ell}^{2}} \leq 1$

The Winter curve for plate buckling, adopted in EN 1993-1-5 (CEN 2006), is given by:

$$
\rho=\left\{\begin{array}{cc}
1 & \text { for } \quad \bar{\lambda}_{\ell} \leq 0.673 \\
\frac{1}{\bar{\lambda}_{\ell}}-\frac{0.22}{\bar{\lambda}_{\ell}^{2}} & \text { for } \quad \bar{\lambda}_{\ell}>0.673
\end{array}\right.
$$

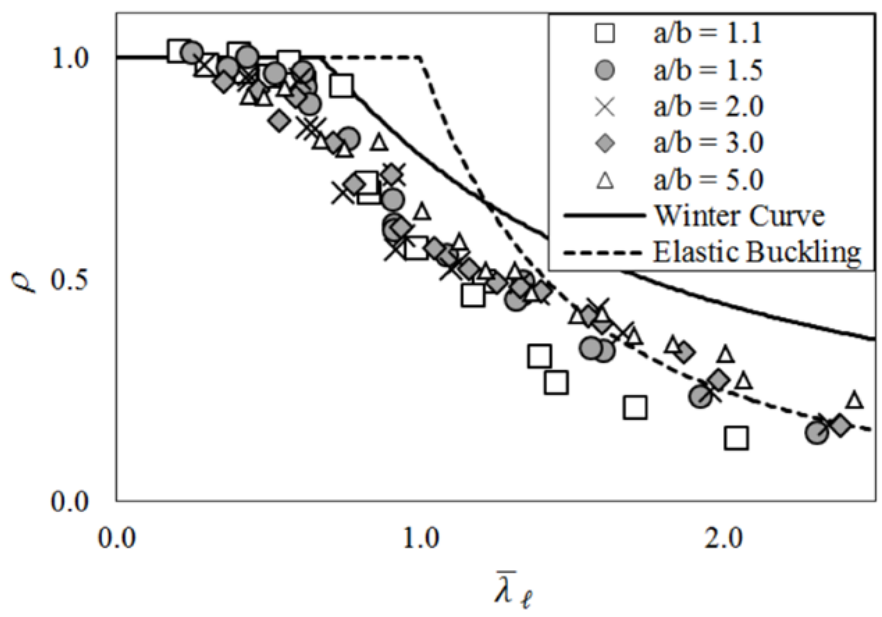

Figure 4. Strength reduction ratios for different aspect ratios

In Figure 4, comparisons are made between the strength reduction $\left(f_{\mathrm{u}} / f_{\mathrm{y}}\right)$ results from the parametric study for the various aspect ratios, the plate buckling Winter curve (shown by the solid line), the elastic buckling curve (shown by the dashed lines). In the interests of clarity, the results for Class B imperfections are plotted in order to demonstrate more clearly the increased imperfection sensitivity displayed by EHS with lower aspect ratios. In Figure 4, it can be observed that as the slenderness increases, the strength of the sections with higher aspect ratios, e.g. $a / b=5.0$ (white triangles), is maintained more effectively than those with lower aspect ratios, e.g. $a / b=$ 1.1 (white boxes). This is an indication that the behaviour of the elliptical sections tends towards that of a plate (represented by the Winter curve) with increasing aspect ratio.

While it can be seen in Figure 4 that the local buckling reduction factors for the EHS with high aspect ratio $(a / b=5)$ is indeed higher than those with low aspect ratios, the results still fall short of the 
Winter curve. It is likely that, for closer convergence to be achieved, the aspect ratio of the EHS member would have to become unrealistically high, such that the geometry of the ellipse along the major axis would more closely approximate the zero-curvature of a flat plate. Nonetheless, the improving postbuckling stability with increasing $a / b$ ratios is clear.

\section{PROPOSED DESIGN METHOD}

Given that expressions for the design of Class 4 EHS do not exist at present, one possible approach to extending the provisions of EN 1993-1-6 (CEN 2007) to EHS members is to apply the equivalent diameter concept, where the diameter $D$ in the design formulae is replaced with $D_{\text {eq }}$ from Eq.(4). However, this approach neglects the stable postbuckling response and reduced imperfection sensitivity exhibited by sections with higher aspect ratios. In this section, the results of the main parametric study are used to define design strength reduction curves for Class 4 EHS in compression. The strength reduction curves were calibrated for each of the aspect ratios examined in the study, using the existing CHS design rules from EN 1993-1-6 (CEN 2007) as a basis, and are given in Eqs.(8) to (16).

$$
\rho=\left\{\begin{array}{cccc}
1 & \text { for } & & \bar{\lambda}_{\ell} \leq \bar{\lambda}_{0} \\
\rho_{1} & \text { for } & \bar{\lambda}_{0}<\bar{\lambda}_{\ell}<\bar{\lambda}_{\mathrm{p}} \\
\rho_{2} & \text { for } & \bar{\lambda}_{\ell} \geq \bar{\lambda}_{\mathrm{p}}
\end{array}\right.
$$

where:

$$
\begin{aligned}
& \bar{\lambda}_{0}=0.288 \\
& \alpha^{\prime}=\frac{0.62}{1+1.91\left(\frac{\Delta w}{(a / b) t}\right)^{1.44}} \\
& \bar{\lambda}_{\mathrm{p}}=\sqrt{\frac{\alpha^{\prime}}{0.4}+\left(\bar{\lambda}_{0}-\sqrt{\frac{\alpha^{\prime}}{0.4}}\right)\left(1-\frac{1}{(a / b)^{0.1}}\right)} \\
& \mu=1-\frac{\beta_{1}}{\bar{\lambda}_{\mathrm{p}}}-\frac{\beta_{2}}{\bar{\lambda}_{\mathrm{p}}^{2}} \\
& \beta_{1}=1-\frac{1}{(a / b)^{0.5}} \\
& \beta_{2}=\frac{0.09+\alpha^{\prime}}{(a / b)}-0.09 \\
& \rho_{1}=1-\mu \frac{\bar{\lambda}_{\ell}-\bar{\lambda}_{0}}{\bar{\lambda}_{\mathrm{p}}-\bar{\lambda}_{0}} \\
& \rho_{2}=\frac{\beta_{1}}{\bar{\lambda}_{\ell}}+\frac{\beta_{2}}{\bar{\lambda}_{\ell}{ }^{2}}
\end{aligned}
$$

The key features of the proposed design curves are: i) a cross-sectional slenderness limit of 90 corresponds to a limiting local buckling slenderness of $\lambda_{\ell}=0.288$, therefore for local buckling slendernesses less than 0.288 , the sections can be assumed to be fully effective and $\rho=1$; ii) similarly to the CHS design curves, the proposed formulae contain a linear portion $\rho_{1}$ and a curved portion $\rho_{2}$, with the range of the linear portion diminishing with increasing $a / b$; iii) the modified imperfection factor $\alpha$ takes into account the reduced imperfection sensitivity of sections with higher aspect ratios. A summary of the design curves for the five aspect ratios examined in the present study is provided in Figure 5 for Class A imperfections, which more closely represent EHS sections in practice.

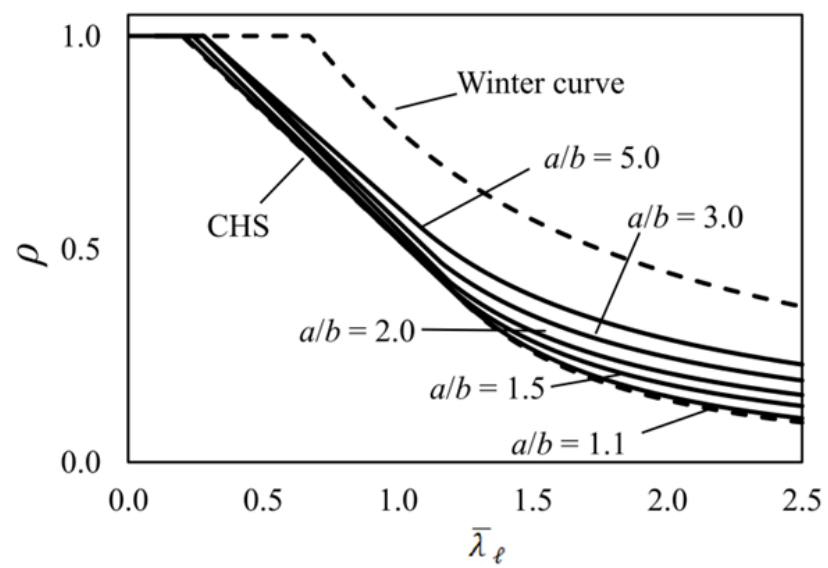

Figure 5. Proposed design curves for slender EHS in compression (Class A imperfections)

When assessing the ratios of ultimate stress as determined from the numerical model $f_{\mathrm{u}, \mathrm{FE}}$ to the design ultimate stress $f_{\mathrm{u}, \mathrm{D}}$ for the proposed EHS design method, it is found that the proposed EHS design method provides safe and accurate predictions for all cases bar one, with values of $f_{\mathrm{u}, \mathrm{FE}} / f_{\mathrm{u}, \mathrm{D}}<1.8$. The direct use of EN 1993-1-6 (CEN 2007) with an equivalent CHS diameter leads to increasingly conservative predictions with increasing aspect ratio and slenderness, with a maximum value of $f_{\mathrm{u}, \mathrm{FE}} / f_{\mathrm{u}, \mathrm{D}}$ of 5.6 for $a / b=5.0$. The relative accuracy of the two methods is demonstrated in Figure 6 for an aspect ratio $a / b=2.0$. Further discussion of the accuracy of the proposed design method is provided in McCann et al. (2016).

\section{CONCLUSIONS}

The local buckling and ultimate strength of Class 4 elliptical hollow sections (EHS) in compression have been investigated using numerical methods. Numerical models were first validated against previous experimental results, after which an extensive 
parametric study was conducted. The parametric study included elliptical sections with aspect ratios from 1.1 to 5.0 , nondimensionalised local buckling slendernesses from 0.21 to 2.49 and wall thicknesses from $0.7 \mathrm{~mm}$ to $8.7 \mathrm{~mm}$. Three different imperfection classes were also considered: $\Delta w=0.1 t$, Class A imperfections and Class B imperfections.

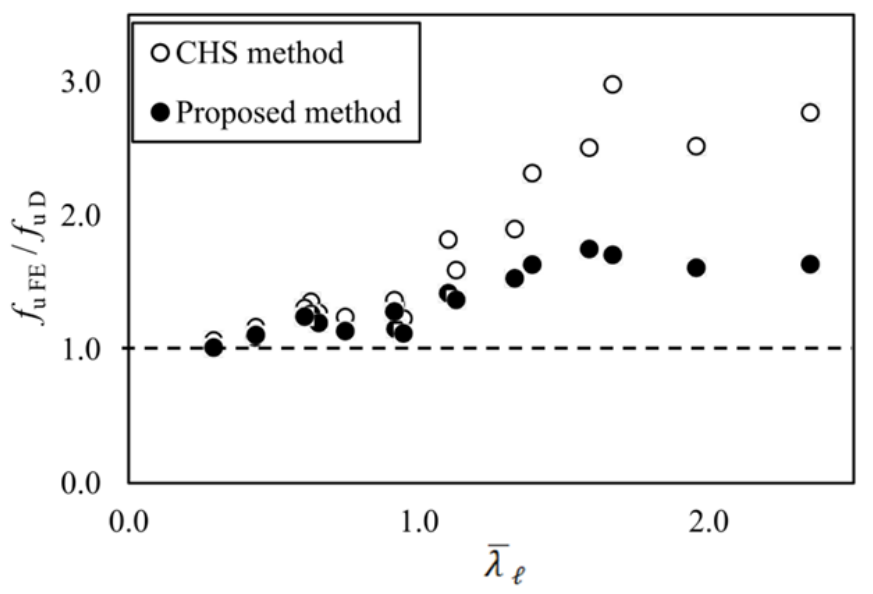

Figure 6. Comparison of equivalent CHS diameter and newproposed design methods for $a / b=2.0$

The EHS with low aspect ratios displayed behaviour more akin to cylindrical shells, with unstable postbuckling response and noticeable imperfection sensitivity, while sections with higher aspect ratios exhibited more plate-like behaviour, with ultimate strengths often exceeding the elastic buckling stress. A general corresponding transition in failure mechanisms was also observed, changing from elephant foot and Yoshimura modes for low aspect ratios to flip-disc and split flip-disc modes for high aspect ratios.

Finally, a design method has been proposed for Class 4 EHS members that reflects the reduction in capacity due to local buckling with increasing slenderness, but also recognises the improved postbuckling stability with increasing aspect ratio. The proposals were shown to provide safe and accurate predictions for the strengths of the EHS columns with nondimensional local slendernesses up to 2.5 and aspect ratios from 1.1 to 5.0. The approach of using an equivalent diameter with the Class 4 CHS design curves from EN 1993-1-6 (CEN 2007) was found to fail to capture the improving postbuckling stability with increasing aspect ratio and thus led to overly conservative results.

\section{ACKNOWLEDGMENTS}

The authors gratefully acknowledge the financial support given by FCT, through IDMEC, under LAETA, project UID/EMS/50022/2013. The authors also wish to recognise the considerable assistance of Mr. Hammad El-Jisr of Imperial College London in the numerical study.

\section{REFERENCES}

ABAQUS 2012. ABAQUS Analysis User's manual, v6.12.3, USA: Dassault Systems Simulia Corp., Providence.

Abela, J.M. \& Gardner, L. 2012. Elastic buckling of elliptical tubes subjected to generalised linearly varying stress distributions. Thin-Walled Structures 58: 40-50.

Chan, T.M. \& Gardner, L. 2008a. Compressive resistance of hot-rolled elliptical hollow sections. Engineering Structures 30(2): 522-532.

Chan, T.M. \& Gardner, L. 2008b. Bending strength of hotrolled elliptical hollow sections. Journal of Constructional Steel Research 64(9): 971-986.

Chan, T.M., Gardner, L. \& Law, K.H. 2010. Structural design of elliptical hollow sections: a review. Proceedings of the Institution of Civil Engineers - Structures and Buildings 163(6): 391-402.

Comité Européen de Normalisation. 2005. EN 1993-1-1:2005 Eurocode 3 - Design of steel structures, Part 1.1: General rules and rules for buildings. London: British Standards Institute.

Comité Européen de Normalisation. 2006. EN 1993-1-5:2006 Eurocode 3 - Design of steel structures, Part 1.5: Plated structural elements. London: British Standards Institute.

Comité Européen de Normalisation. 2007. EN 1993-1-6:2007 Eurocode 3 - Design of steel structures, Part 1.6: General rules - Strength and stability of shell structures. London: British Standards Institute.

Gardner, L. \& Chan, T.M. 2007. Cross-section classification of elliptical hollow sections. Steel and Composite Structures 7(3):185-200.

Insausti, A. \& Gardner, L. 2011. Analytical modelling of plastic collapse in compressed elliptical hollow sections. Journal of Constructional Steel Research 67: 678-89.

Kempner, J. \& Chen, Y.N. 1964. Large deflections of an axially compressed oval cylindrical shell. Proceedings of the 11th International Congress of Applied Mechanics, Munich, 1964.

Law, K.H. \& Gardner, L. 2013. Buckling of elliptical hollow section members under combined compression and uniaxial bending. Journal of Constructional Steel Research 86: 116.

McCann, F., Gardner, L. \& Qiu, W. 2015. Experimental study of slender concrete-filled elliptical hollow section beamcolumns. Journal of Constructional Steel Research 113: 185-194.

McCann, F., Fang, C., Gardner, L. \& Silvestre, N. 2015. Local buckling and ultimate strength of slender elliptical hollow sections in compression. Engineering Structures 111: 104118.

Murray, N. 1984. Introduction to the theory of thin-walled structures. Oxford: Oxford University Press.

Silvestre, N. 2008. Buckling behaviour of elliptical cylindrical shells and tubes under compression. International Journal of Solids and Structures 45(16): 4427-4447.

Silvestre, N. \& Gardner, L. 2011. Elastic local postbuckling of elliptical tubes. Journal of Constructional Steel Research 67(3): 281-292.

Ruiz-Teran, A.M. \& Gardner, L. 2008. Elastic buckling of elliptical tubes. Thin-Walled Structures 46(11):1304-1318.

Steel Construction Institute. 2013. Steel building design: Design data. Publication Number P363. Ascot: SCI.

Theofanous, M., Chan, T.M. \& Gardner, L. 2009. Structural response of stainless steel oval hollow section compression members. Engineering Structures 31(4): 922-34.

Zhu, J.H. \& Young, B. 2012. Design of cold-formed steel oval hollow section columns. Journal of Constructional Steel Research 71: 26-37. 